PROCEEDINGS OF THE

AMERICAN MATHEMATICAL SOCIETY

Volume 139, Number 3, March 2011, Pages 917-930

S 0002-9939(2010)10511-7

Article electronically published on October 29, 2010

\title{
INEQUIVALENT MEASURES OF NONCOMPACTNESS AND THE RADIUS OF THE ESSENTIAL SPECTRUM
}

\author{
JOHN MALLET-PARET AND ROGER D. NUSSBAUM
}

(Communicated by Nigel J. Kalton)

Abstract. The Kuratowski measure of noncompactness $\alpha$ on an infinite dimensional Banach space $(X,\|\cdot\|)$ assigns to each bounded set $S$ in $X$ a nonnegative real number $\alpha(S)$ by the formula

$$
\begin{aligned}
\alpha(S)= & \inf \left\{\delta>0 \mid S=\bigcup_{i=1}^{n} S_{i} \text { for some } S_{i}\right. \\
& \text { with } \left.\operatorname{diam}\left(S_{i}\right) \leq \delta, \text { for } 1 \leq i \leq n<\infty\right\} .
\end{aligned}
$$

In general a map $\beta$ which assigns to each bounded set $S$ in $X$ a nonnegative real number and which shares most of the properties of $\alpha$ is called a homogeneous measure of noncompactness or homogeneous MNC. Two homogeneous MNC's $\beta$ and $\gamma$ on $X$ are called equivalent if there exist positive constants $b$ and $c$ with $b \beta(S) \leq \gamma(S) \leq c \beta(S)$ for all bounded sets $S \subset X$. There are many results which prove the equivalence of various homogeneous MNC's. Working with $X=\ell^{p}(\mathbb{N})$ where $1 \leq p \leq \infty$, we give the first examples of homogeneous MNC's which are not equivalent.

Further, if $X$ is any complex, infinite dimensional Banach space and $L$ : $X \rightarrow X$ is a bounded linear map, one can define $\rho(L)=\sup \{|\lambda| \mid \lambda \in \operatorname{ess}(L)\}$, where $\operatorname{ess}(L)$ denotes the essential spectrum of $L$. One can also define

$$
\beta(L)=\inf \{\lambda>0 \mid \beta(L S) \leq \lambda \beta(S) \text { for every } S \in \mathcal{B}(X)\} .
$$

The formula $\rho(L)=\lim _{m \rightarrow \infty} \beta\left(L^{m}\right)^{1 / m}$ is known to be true if $\beta$ is equivalent to $\alpha$, the Kuratowski MNC; however, as we show, it is in general false for MNC's which are not equivalent to $\alpha$. On the other hand, if $B$ denotes the unit ball in $X$ and $\beta$ is any homogeneous MNC, we prove that

$$
\rho(L)=\limsup _{m \rightarrow \infty} \beta\left(L^{m} B\right)^{1 / m}=\inf \left\{\lambda>0 \mid \lim _{m \rightarrow \infty} \lambda^{-m} \beta\left(L^{m} B\right)=0\right\} .
$$

Our motivation for this study comes from questions concerning eigenvectors of linear and nonlinear cone-preserving maps.

If $(X, d)$ is a complete metric space and $S$ is a bounded subset of $X$, then K. Kuratowski [10 has defined $\alpha(S)$, the Kuratowski measure of noncompactness of $S$, by

$$
\alpha(S):=\inf \left\{\delta>0 \mid S=\bigcup_{i=1}^{n} S_{i} \text { for some } S_{i} \text { with } \operatorname{diam}\left(S_{i}\right) \leq \delta, \text { for } 1 \leq i \leq n<\infty\right\} .
$$

Received by the editors September 21, 2009 and, in revised form, January 16, 2010.

2010 Mathematics Subject Classification. Primary 47H08, 46B20; Secondary 46B25, 46B45, 47A10, 47H10.

Key words and phrases. Measure of noncompactness, essential spectral radius, cone map.

The first author was partially supported by NSF Grant DMS-0500674.

The second author was partially supported by NSF Grant DMS-0701171.

(C)2010 American Mathematical Society 
Here $\operatorname{diam}(T)$ denotes the diameter of a set $T \subset X$, namely

$$
\operatorname{diam}(T):=\sup \{d(x, y) \mid x, y \in T\} .
$$

We shall denote by $\mathcal{B}(X)$ the collection of all bounded subsets of $X$. Kuratowski has shown, and it is straightforward to verify, that $\alpha$ satisfies the following properties:

(K1) $\alpha(S)=0$ if and only if $\bar{S}$ is compact, for every $S \in \mathcal{B}(X)$;

(K2) $\alpha(S) \leq \alpha(T)$ for every $S, T \in \mathcal{B}(X)$ with $S \subset T$;

(K3) $\alpha\left(S \cup\left\{x_{0}\right\}\right)=\alpha(S)$ for every $S \in \mathcal{B}(X)$ and $x_{0} \in X$; and

(K4) $\alpha(\bar{S})=\alpha(S)$ for every $S \in \mathcal{B}(X)$.

If $S$ and $T$ are subsets of a real or complex Banach space $(X,\|\cdot\|)$ and $\lambda$ is a scalar, we shall let $\operatorname{co}(S)$ denote the convex hull of $S$, namely the smallest convex set containing $S$, and we shall write $S+T:=\{s+t \mid s \in S$ and $t \in T\}$ and $\lambda S:=\{\lambda s \mid s \in S\}$. G. Darbo [6] has observed that, assuming the metric on $X$ is the usual one obtained from the norm $\|\cdot\|$, the following properties hold:

(K5) $\alpha(\operatorname{co}(S))=\alpha(S)$ for every $S \in \mathcal{B}(X)$;

(K6) $\alpha(S+T) \leq \alpha(S)+\alpha(T)$ for every $S, T \in \mathcal{B}(X)$; and

(K7) $\alpha(\lambda S)=|\lambda| \alpha(S)$ for every $S \in \mathcal{B}(X)$ and every scalar $\lambda$.

Properties (K5), (K6), and (K7) make the Kuratowski MNC a very useful tool in fixed point theory and functional analysis. Let us also mention the following so-called set-additivity property, which holds in any metric space:

(K8) $\alpha(S \cup T)=\max \{\alpha(S), \alpha(T)\}$ for every $S, T \in \mathcal{B}(X)$.

If $(X,\|\cdot\|)$ is a real or complex Banach space, we shall say that a map $\beta: \mathcal{B}(X) \rightarrow$ $[0, \infty)$ is a homogeneous measure of noncompactness on $\mathbf{X}$ or homogeneous MNC if $\beta$ satisfies properties (K1)-(K7), with $\beta$ replacing $\alpha$ in these conditions. We shall say that $\beta$ is a homogeneous, set-additive $\mathbf{M N C}$ if $\beta$ satisfies properties (K1)-(K8), with $\beta$ replacing $\alpha$ in these conditions. Our terminology differs from some of the literature [1, 2], [3, [18, where a map satisfying properties (K1)-(K8) is simply called an MNC. Of course these properties are not independent. For example, properties (K2), (K6), and (K7) imply property (K4).

If $\beta$ and $\gamma$ are homogeneous MNC's on $X$, we say that $\boldsymbol{\beta}$ dominates $\gamma$ if there exists a number $c>0$ such that $\gamma(S) \leq c \beta(S)$ for every $S \in \mathcal{B}(X)$. If $\beta$ and $\gamma$ are homogeneous MNC's on $X$ such that both $\beta$ dominates $\gamma$ and $\gamma$ dominates $\beta$, we say that $\beta$ and $\gamma$ are equivalent. There are many examples of homogeneous MNC's (see [1, 2], 3], 44, 14, 15, 16, 17, 18]), but up to now all known examples of homogeneous MNC's on a given Banach space $X$ are equivalent. This fact begs the following question.

Question A. Does there exist a Banach space $(X,\|\cdot\|)$ for which there is a homogeneous (possibly set-additive) MNC $\beta$ on $X$ which is not equivalent to the Kuratowski MNC $\alpha$ on $X$ ?

As we shall see below in Theorem 17, where a class of inequivalent MNC's is constructed, Question A is answered in the affirmative.

If $L: X \rightarrow X$ is a bounded linear map and $\beta$ is a homogeneous MNC on $X$, one can define

$$
\begin{aligned}
& \beta(L):=\inf \{\lambda \geq 0 \mid \beta(L S) \leq \lambda \beta(S) \text { for every bounded } S \subset X\}, \\
& \beta^{\#}(L):=\limsup _{m \rightarrow \infty} \beta\left(L^{m}\right)^{1 / m},
\end{aligned}
$$


where we set $\beta(L)=\infty$ if the set in the first line of (11) is empty. If it is in fact the case that $\beta(L)<\infty$, then one easily shows that

$$
\beta^{\#}(L)=\lim _{m \rightarrow \infty} \beta\left(L^{m}\right)^{1 / m}=\inf _{m \geq 1} \beta\left(L^{m}\right)^{1 / m},
$$

which follows directly from the fact that $\beta\left(L^{m+n}\right) \leq \beta\left(L^{m}\right) \beta\left(L^{n}\right)<\infty$ for every $m \geq 1$ and $n \geq 1$. Lemma 4 below implies that if $\beta$ is equivalent to the Kuratowski MNC $\alpha$ on $X$, then there exists a constant $c>0$, independent of $L$, with $\beta(L) \leq$ $c \alpha(L) \leq c\|L\|<\infty$. Additionally, if $\beta$ is equivalent to $\alpha$, the results of [14] imply that $\beta^{\#}(L)=\rho(L)$, where $\rho(L)$ denotes the radius of the essential spectrum of $L$. This suggests the following question.

Question B. Is it the case that $\beta^{\#}(L)=\rho(L)$ for any homogeneous MNC $\beta$ on $X$, where $\rho(L)$ denotes the radius of the essential spectrum of $L$ ? If this is not the case, is there an analogous formula for $\rho(L)$ which holds for any homogeneous $\mathrm{MNC} \beta$ ?

For a general homogeneous MNC $\beta$ which is not equivalent to $\alpha$, we shall establish in Theorem 8 below that it may happen that $\beta^{\#}(L) \neq \rho(L)$, and in fact it may happen that $\beta\left(L^{m}\right)=\infty$ for all $m \geq 1$. Elsewhere [13], we shall construct an example for which

$$
\liminf _{m \rightarrow \infty} \beta\left(L^{m}\right)^{1 / m}<\limsup _{m \rightarrow \infty} \beta\left(L^{m}\right)^{1 / m}=\infty .
$$

In such cases $\beta^{\#}(L)=\infty$ while $\rho(L)<\infty$. As will be shown in Theorem 10 below, in place of the quantity $\beta^{\#}(L)$ the appropriate quantity to consider is

$$
\beta^{*}(L):=\limsup _{m \rightarrow \infty} \beta\left(L^{m} B_{1}(0)\right)^{1 / m}=\inf \left\{\lambda>0 \mid \lim _{m \rightarrow \infty} \lambda^{-m} \beta\left(L^{m} B_{1}(0)\right)=0\right\},
$$

as it is the case that $\beta^{*}(L)=\rho(L)$ for every homogeneous MNC $\beta$ and every bounded linear operator $L$ on $X$. We denote

$$
B_{r}(x):=\{y \in X \mid\|y-x\|<r\}
$$

both here and below.

Remark. In order for $\rho(L)$ to be defined above, one needs to have a linear operator on a complex Banach space. Suppose instead that $X$ is a real Banach space, $\beta$ is a homogeneous MNC on $X$, and $L: X \rightarrow X$ is a bounded linear map. The complexification $\widehat{X}$ of $X$ equals $\{(u, v) \mid u, v \in X\}$. If one identifies $(u, v)$ with $u+i v$ where $i^{2}=-1$, and defines

$$
\|u+i v\|:=\sup _{0 \leq \theta \leq 2 \pi}\|(\cos \theta) u+(\sin \theta) v\|,
$$

then $\widehat{X}$ becomes a complex Banach space. The linear map $L$ then extends to a complex linear map $\widehat{L}$ on $\widehat{X}$ by $\widehat{L}(u+i v)=L u+i L v$. It is also the case that $\beta$ extends to a homogeneous MNC $\widehat{\beta}$ on $\widehat{X}$ as follows. For $x=u+i v \in \widehat{X}$ define $\operatorname{Re}(x):=u$, and for $\widehat{S} \in \mathcal{B}(\widehat{X})$ define $\operatorname{Re}(\widehat{S}):=\{\operatorname{Re}(x) \mid x \in \widehat{S}\}$ and set

$$
\widehat{\beta}(\widehat{S}):=\sup _{0 \leq \theta \leq 2 \pi} \beta\left(\operatorname{Re}\left(e^{i \theta} \widehat{S}\right)\right) .
$$

One can prove that $\widehat{\beta}$ is a homogeneous MNC on the complex Banach space $\widehat{X}$, that $\widehat{\beta}\left(\widehat{L}^{m}\right)=\beta\left(L^{m}\right)$, and that $\widehat{\beta}\left(\widehat{L}^{m} \widehat{B}_{1}(0)\right)=\beta\left(L^{m} B_{1}(0)\right)$, where $\widehat{B}_{1}(0)$ (respectively, 
$\left.B_{1}(0)\right)$ denotes the unit ball in $\widehat{X}$ (respectively, $X$ ). It follows that

$$
\widehat{\beta}^{\#}(\widehat{L})=\beta^{\#}(L), \quad \widehat{\beta}^{*}(\widehat{L})=\beta^{*}(L)
$$

both hold. We remark also that if $\alpha$ denotes the Kuratowski MNC on a real Banach space $X$ and $\widehat{\alpha}$ denotes its complexification as above, then $\widehat{\alpha}$ is in fact the Kuratowski MNC on $\widehat{X}$. We omit the proofs of these results, which are straightforward for the most part, except for the proof that $\widehat{\alpha}$ is the Kuratowski MNC on $\widehat{X}$; this is given as Proposition 11.

Our interest in Questions A and B and the related issues above arises from the question of the "correct" definition of the "cone essential spectral radius," denoted $\rho_{C}(f)$, for a map $f: C \rightarrow C$. Here $C$ is a closed cone in a Banach space and $f$ is a continuous, homogeneous, order-preserving map. This question is, in turn, related to the problem of existence of an eigenvector of $f$ in $C$ with eigenvalue equal to $r_{C}(f)$, the "cone spectral radius of $f$," and to showing that $\rho_{C}(f) \leq r_{C}(f)$; see [11] and [17. In future work, related to this paper, we shall discuss deficiencies in the definition of $\rho_{C}(f)$ in [11, [17, and theorems about existence of eigenvectors of $f$.

Theorems 7, 8, and 10 are the main results of this paper. In Theorem 7 we shall present the first known example of an infinite dimensional Banach space $Y=\ell^{p}(\mathbb{N})$ and a homogeneous, set-additive MNC $\gamma_{Y}$ on $Y$ which is not equivalent to the Kuratowski MNC, thereby answering Question A in the affirmative. In fact, we provide a large class of such inequivalent MNC's $\gamma_{Y}$. Much more general results for other spaces are given in [12, but it seems worthwhile to illustrate our approach here in this relatively simple case with a self-contained proof. (In fact we use some ideas from [12] in the example considered in Theorem 8,) In Theorem 8 we study the quantities $\gamma_{Z}\left(\Lambda^{m}\right)$ and $\gamma_{Z}^{\#}(\Lambda)$ for homogeneous, set-additive MNC's $\gamma_{Z}$ on $Z=\ell^{p}(\mathbb{N} \times \mathbb{N})$ related to the MNC's $\gamma_{Y}$ of Theorem 7 , for a particular shift operator $\Lambda$ on the space $Z$. We demonstrate the pathological features of these quantities noted above, in particular that in general $\gamma_{Z}^{\#}(\Lambda) \neq \rho(\Lambda)$, which thereby gives a negative answer to the first part of Question B. In Theorem 10 we prove for a general homogeneous MNC $\beta$ on a Banach space $X$ that $\beta^{*}(L)$ rather than $\beta^{\#}(L)$ is the "correct" quantity to consider in studying $\rho(L)$. In particular we show that $\beta^{*}(L)=\rho(L)$ always holds for all bounded linear operators on $X$, thus providing an affirmative answer to the second part of Question B.

Due to the following result proved in [12, the issue of whether or not a homogeneous MNC satisfies the set-additivity property (K8) is often unimportant.

Proposition 1 (see [12]). Let $(X,\|\cdot\|)$ be a Banach space and $\beta$ a homogeneous $M N C$ on $X$. For $S \in \mathcal{B}(X)$, define $\gamma(S)$ by

$$
\gamma(S):=\inf \left\{\max _{1 \leq i \leq n} \beta\left(S_{i}\right) \mid S=\bigcup_{i=1}^{n} S_{i} \text { for some } S_{i} \text { with } 1 \leq i \leq n<\infty\right\} .
$$

Then $\gamma$ is a homogeneous, set-additive $M N C$ on $X$ with $\gamma(S) \leq \beta(S)$ for all bounded $S \subset X$. Moreover, $\gamma=\beta$ if $\beta$ itself is a homogeneous, set-additive MNC.

Before presenting our main results we make some fundamental observations.

Proposition 2. Let $(X,\|\cdot\|)$ be a Banach space and $\beta$ a homogeneous $M N C$ on $X$. Then the Kuratowski $M N C \alpha$ dominates $\beta$. 
Proof. Let $c:=\beta\left(B_{1}(0)\right)$, recalling the notation (4). Then homogeneity implies that $\beta\left(B_{r}(0)\right)=r c$. If $S \in \mathcal{B}(X)$ and $d:=\alpha(S)$, then given $\varepsilon>0$, there exists a finite collection of sets $S_{1}, S_{2}, \ldots, S_{n}$ with $S=\bigcup_{i=1}^{n} S_{i}$, and with $\operatorname{diam}\left(S_{i}\right) \leq d+\varepsilon$ for $1 \leq i \leq n$. For each $i$ select $x_{i} \in S_{i}$ and define $T:=\left\{x_{i} \mid 1 \leq i \leq n\right\}$. Note that $S \subset T+B_{d+\varepsilon}(0)$, so property (K6), along with (K1) and (K2), implies that

$$
\beta(S) \leq \beta(T)+\beta\left(B_{d+\varepsilon}(0)\right)=\beta\left(B_{d+\varepsilon}(0)\right)=(d+\varepsilon) c .
$$

Since $\varepsilon>0$ is arbitrary, we conclude that $\beta(S) \leq c d=c \alpha(S)$.

The next result was obtained independently by Furi and Vignoli in [7] and by Nussbaum in Section A of [16].

Proposition 3 (see [7] and Section A of [16]). Let $(X,\|\cdot\|)$ be an infinite dimensional Banach space. If $Q:=\{x \in X \mid\|x\| \leq 1\}$ and if $\alpha$ denotes the Kuratowski $M N C$ on $X$, then $\alpha(Q)=2$.

Lemma 4 below is an easy result; see [14] or Section A of [16]. However, as we shall see later, Lemma 4 may fail for general homogeneous MNC's.

Lemma 4 (see [14] or Section A of [16]). Let $\left(X_{i},\|\cdot\|_{i}\right)$, for $i=1,2$, be Banach spaces, let $\alpha_{i}$ denote the Kuratowski $M N C$ on $X_{i}$, and let $L: X_{1} \rightarrow X_{2}$ be a bounded linear map. Define

$$
\alpha(L):=\inf \left\{\lambda \geq 0 \mid \alpha_{2}(L S) \leq \lambda \alpha_{1}(S) \text { for every bounded } S \subset X_{1}\right\} .
$$

Then we have $\alpha(L) \leq\|L\|$. Further, if $\beta_{i}$ is a homogeneous $M N C$ on $X_{i}$, with $\beta_{i}$ equivalent to $\alpha_{i}$ for $i=1,2$, then there exists a constant $c>0$, independent of $L$, such that

$$
\beta_{2}(L S) \leq c \alpha(L) \beta_{1}(S) \leq c\|L\| \beta_{1}(S)
$$

for every $S \in \mathcal{B}\left(X_{1}\right)$.

Our next lemma is true in greater generality (see [12]), but the following version will suffice for our purposes.

Lemma 5. Let $\left(X_{i},\|\cdot\|_{i}\right)$, for $i=1,2$, be Banach spaces, and let $L: X_{1} \rightarrow X_{2}$ be a one-one, continuous linear map of $X_{1}$ onto $X_{2}$. If $\beta_{2}$ is a homogeneous MNC on $X_{2}$, define, for $S \in \mathcal{B}\left(X_{1}\right)$,

$$
\widetilde{\beta}_{2}(S):=\beta_{2}(L S) .
$$

Then $\widetilde{\beta}_{2}$ is a homogeneous $M N C$ on $X_{1}$, and $\widetilde{\beta}_{2}$ is set-additive if $\beta_{2}$ is set-additive. If $\alpha_{i}$ denotes the Kuratowski MNC on $X_{i}$ and if $\beta_{2}$ is equivalent to $\alpha_{2}$, then $\widetilde{\beta}_{2}$ is equivalent to $\alpha_{1}$.

Proof. The fact that $\widetilde{\beta}_{2}$ is a homogeneous (set-additive) MNC on $X_{1}$ follows easily from the fact that $L$ is a linear homeomorphism of $X_{1}$ onto $X_{2}$. Details are left to the reader.

To see that $\widetilde{\beta}_{2}$ is equivalent to $\alpha_{1}$ if $\beta_{2}$ is equivalent to $\alpha_{2}$, observe that $\widetilde{\beta}_{2}$ is equivalent to $\widetilde{\alpha}_{2}$, where $\widetilde{\alpha}_{2}(S):=\alpha_{2}(L S)$. Thus it suffices to prove that $\widetilde{\alpha}_{2}$ is equivalent to $\alpha_{1}$. However, if $S$ is a bounded subset of $X_{1}$, then Lemma 4 implies that $\alpha_{2}(L S) \leq\|L\| \alpha_{1}(S)$ and $\alpha_{1}(S)=\alpha_{1}\left(L^{-1} L S\right) \leq\left\|L^{-1}\right\| \alpha_{2}(L S)$. This proves that $\widetilde{\alpha}_{2}$ and $\alpha_{1}$ are equivalent.

The following lemma will be convenient in establishing Theorem 7 . 
Lemma 6. Let $\left(X_{i},\|\cdot\|_{i}\right)$, for $i=1,2$, be Banach spaces, let $\alpha_{i}$ denote the Kuratowski $M N C$ on $X_{i}$, and let $L: X_{1} \rightarrow X_{2}$ be a one-one, continuous linear map of $X_{1}$ onto $X_{2}$. Suppose there exists a homogeneous $M N C \beta_{2}$ on $X_{2}$ which is inequivalent to $\alpha_{2}$. Then there exists a homogeneous, set-additive $M N C \gamma_{2}$ on $X_{2}$ which is inequivalent to $\alpha_{2}$. Further, there exists a homogeneous, set-additive $M N C \gamma_{1}$ on $X_{1}$ which is inequivalent to $\alpha_{1}$.

Proof. Proposition 2 implies that $\alpha_{2}$ dominates $\beta_{2}$, so there must exist a sequence of bounded sets $S_{n} \subset X_{2}$ with $\alpha_{2}\left(S_{n}\right)>0$ and $\lim _{n \rightarrow \infty} \frac{\beta_{2}\left(S_{n}\right)}{\alpha_{2}\left(S_{n}\right)}=0$. Let $\gamma_{2}$ be the homogeneous, set-additive MNC derived from $\beta_{2}$ as in Proposition 1, Then it is immediate that $\gamma_{2}(S) \leq \beta_{2}(S)$ for all $S \in \mathcal{B}\left(X_{2}\right)$, and so $\lim _{n \rightarrow \infty} \frac{\gamma_{2}\left(S_{n}\right)}{\alpha_{2}\left(S_{n}\right)}=0$. Define $\widetilde{\gamma}_{2}$ and $\widetilde{\alpha}_{2}$ as in Lemma 5 , so $\widetilde{\gamma}_{2}(T):=\gamma_{2}(L T)$ and $\widetilde{\alpha}_{2}(T):=\alpha_{2}(L T)$ for $T \in \mathcal{B}\left(X_{1}\right)$. Then Lemma 5 implies that $\widetilde{\gamma}_{2}$ and $\widetilde{\alpha}_{2}$ are homogeneous, set-additive MNC's on $X_{1}$ and that $\widetilde{\alpha}_{2}$ is equivalent to $\alpha_{1}$, so in particular there exists $c>0$ such that $\widetilde{\alpha}_{2}(T) \leq c \alpha_{1}(T)$ for every $T \in \mathcal{B}\left(X_{1}\right)$. If we define $T_{n}:=L^{-1} S_{n}$, it follows that

$$
\lim _{n \rightarrow \infty}\left(\frac{\widetilde{\gamma}_{2}\left(T_{n}\right)}{\alpha_{1}\left(T_{n}\right)}\right) \leq c \lim _{n \rightarrow \infty}\left(\frac{\widetilde{\gamma}_{2}\left(T_{n}\right)}{\widetilde{\alpha}_{2}\left(T_{n}\right)}\right)=c \lim _{n \rightarrow \infty}\left(\frac{\gamma_{2}\left(S_{n}\right)}{\alpha_{2}\left(S_{n}\right)}\right)=0
$$

so $\widetilde{\gamma}_{2}$ and $\alpha_{1}$ are inequivalent. If we define $\gamma_{1}:=\widetilde{\gamma}_{2}$, the proof is complete.

Let $1 \leq p \leq \infty$ and let $\mathbb{N}$ denote the natural numbers. We define the Banach space $Y:=\ell^{p}(\mathbb{N})$ in the usual way: Elements $y \in Y$ are maps $y: \mathbb{N} \rightarrow \mathbb{C}$ such that $\|y\|_{Y}:=\left(\sum_{i=1}^{\infty}|y(i)|^{p}\right)^{1 / p}<\infty$. As usual, we interpret $\|y\|_{Y}:=\sup _{i \in \mathbb{N}}|y(i)|$ if $p=\infty$. (We remark that if we instead take the corresponding real Banach space of maps $y: \mathbb{N} \rightarrow \mathbb{R}$, then the construction below is still valid with the obvious changes.) Similarly, the Banach space $Z:=\ell^{p}(\mathbb{N} \times \mathbb{N})$ is the set of maps $z: \mathbb{N} \times \mathbb{N} \rightarrow \mathbb{C}$ such that $\|z\|_{Z}:=\left(\sum_{i=1}^{\infty} \sum_{j=1}^{\infty}|z(i, j)|^{p}\right)^{1 / p}<\infty$, and again with the corresponding supremum norm if $p=\infty$. It is well-known that there is a oneone map $\sigma: \mathbb{N} \times \mathbb{N} \rightarrow \mathbb{N}$ of $\mathbb{N} \times \mathbb{N}$ onto $\mathbb{N}$, and that $\sigma$ induces a linear isometry $L_{\sigma}: Y \rightarrow Z$ by composition, namely $L_{\sigma} y:=y \circ \sigma$. We want to prove that there is a homogeneous, set-additive MNC $\gamma_{Y}$ on $Y$ which is inequivalent to the Kuratowski MNC $\alpha_{Y}$ on $Y$. By Lemma 6 it suffices to prove that there exists a homogeneous MNC $\beta_{Z}$ on $Z$ which is inequivalent to the Kuratowski MNC $\alpha_{Z}$ on $Z$.

Theorem 7. Let $1 \leq p \leq \infty$ and let $Y$ denote the Banach space $\ell^{p}(\mathbb{N})$ with the usual norm. Let $\alpha_{Y}$ denote the Kuratowski MNC on $Y$. Then there exists a homogeneous, set-additive $M N C \gamma_{Y}$ on $Y$ which is inequivalent to $\alpha_{Y}$.

Proof. With $Z=\ell^{p}(\mathbb{N} \times \mathbb{N})$ and with the norm $\|\cdot\|_{Z}$ as above, let $\alpha_{Z}$ denote the Kuratowski MNC on $Z$. By the remarks above, it suffices to prove that there exists a homogeneous MNC $\beta_{Z}$ on $Z$ which is inequivalent to $\alpha_{Z}$.

For simplicity, we shall denote $\alpha_{Z}$ and $\beta_{Z}$ simply by $\alpha$ and $\beta$, respectively, and we denote $\mathcal{B}:=\mathcal{B}(Z)$, the set of bounded subsets of $Z$. Also for simplicity, in what follows we shall assume that $p<\infty$, as the arguments for $p=\infty$ are similar.

Let $a_{n}$, for $n \geq 1$, be a nonincreasing sequence of positive reals with $a_{1} \leq 1$ and $\lim _{n \rightarrow \infty} a_{n}=0$. Define a Banach space $(\widetilde{Z},\|\cdot\| \widetilde{Z})$ to be the set of maps $z: \mathbb{N} \times \mathbb{N} \rightarrow \mathbb{C}$ such that

$$
\|z\| \widetilde{Z}:=\left(\sum_{i=1}^{\infty} a_{i}^{p} \sum_{j=1}^{\infty}|z(i, j)|^{p}\right)^{1 / p}<\infty
$$


and let $\widetilde{\alpha}$ denote the Kuratowski MNC on $\widetilde{Z}$. Note that $Z \subset \widetilde{Z}$ and that

$$
\|z\|_{\widetilde{Z}} \leq\|z\|_{Z}
$$

for all $z \in Z$. For each integer $n \geq 1$ define the linear projection $P_{n}: Z \rightarrow Z$ by setting $P_{n} z=x$, where

$$
x(i, j)= \begin{cases}z(i, j), & \text { for } 1 \leq i \leq n, \\ 0, & \text { for } i>n .\end{cases}
$$

Note also that $P_{n}: \widetilde{Z} \rightarrow \widetilde{Z}$ is a projection and that $P_{n} \widetilde{Z}=P_{n} Z$. It is easy to see that, for all $z \in Z$,

$$
\left\|P_{n} z\right\|_{Z} \leq\|z\|_{Z}, \quad\left\|P_{n} z\right\|_{\tilde{Z}} \leq\|z\|_{\tilde{Z}}, \quad\left\|P_{n} z\right\|_{Z} \leq a_{n}^{-1}\left\|P_{n} z\right\| \widetilde{Z},
$$

and in fact the second and third inequalities in (9) are valid for every $z \in \widetilde{Z}$. Thus by Lemma 4, using (8) and (9), we have that

$$
\begin{aligned}
& \widetilde{\alpha}(S) \leq \alpha(S), \quad \alpha\left(P_{n} S\right) \leq \alpha(S), \quad \widetilde{\alpha}\left(P_{n} S\right) \leq \widetilde{\alpha}(S), \\
& \alpha\left(P_{n} S\right) \leq a_{n}^{-1} \widetilde{\alpha}\left(P_{n} S\right),
\end{aligned}
$$

for every $S \in \mathcal{B}$. We now define $\mathcal{A} \subset \mathcal{B}$ by

$$
\mathcal{A}:=\left\{S \in \mathcal{B} \mid \lim _{n \rightarrow \infty} \alpha\left(\left(I-P_{n}\right) S\right)=0\right\} .
$$

The reader can easily verify that if $S, T \in \mathcal{A}$ and $\lambda \in \mathbb{C}$, then the sets $\operatorname{co}(S), \lambda S$, $\bar{S}$, and $S+T$ are all elements of $\mathcal{A}$. Furthermore, if $S \in \mathcal{B}$, then $P_{n} S \in \mathcal{A}$ for every integer $n \geq 1$.

With these preliminaries we define $\beta: \mathcal{B} \rightarrow[0, \infty)$ by

$$
\beta(S):=\inf \{\widetilde{\alpha}(A)+\alpha(B) \mid S \subset A+B, \text { for some } A \in \mathcal{A} \text { and } B \in \mathcal{B}\} .
$$

We claim that $\beta$ is a homogeneous MNC on $Z$, that $\beta$ is inequivalent to $\alpha$, and that $\beta(S)=\widetilde{\alpha}(S)$ for all $S \in \mathcal{A}$.

Observe first that for any $S \in \mathcal{B}$, if we take $A:=\{0\}$ and $B:=S$ in equation (12), we see that $\beta(S) \leq \alpha(S)$.

If $S \in \mathcal{A}$ and we take $A:=S$ and $B:=\{0\}$ in (12), we see that $\beta(S) \leq \widetilde{\alpha}(S)$. On the other hand, if $S \in \mathcal{A}$ and $S \subset A+B$, where $A \in \mathcal{A}$ and $B \in \mathcal{B}$, we have, using the first inequality in (10), that

$$
\widetilde{\alpha}(S) \leq \widetilde{\alpha}(A)+\widetilde{\alpha}(B) \leq \widetilde{\alpha}(A)+\alpha(B),
$$

so we obtain from (12) that $\widetilde{\alpha}(S) \leq \beta(S)$. We conclude that $\widetilde{\alpha}(S)=\beta(S)$ for $S \in \mathcal{A}$, as claimed.

The fact that $\beta$ satisfies property (K2) (with $\beta$ replacing $\alpha$ ) is obvious. It follows that if $S \in \mathcal{B}$, then $\beta(S) \leq \beta(\operatorname{co}(S))$. On the other hand, given $\varepsilon>0$, select $A \in \mathcal{A}$ and $B \in \mathcal{B}$ so that $S \subset A+B$ and $\beta(S) \leq \widetilde{\alpha}(A)+\alpha(B)<\beta(S)+\varepsilon$. Note that $\operatorname{co}(A)+\operatorname{co}(B)$ is a convex set containing $S$, so $\operatorname{co}(S) \subset \operatorname{co}(A)+\operatorname{co}(B)$. Since $\operatorname{co}(A) \in \mathcal{A}$, we conclude that

$$
\beta(\operatorname{co}(S)) \leq \widetilde{\alpha}(\operatorname{co}(A))+\alpha(\operatorname{co}(B))=\widetilde{\alpha}(A)+\alpha(B)<\beta(S)+\varepsilon,
$$

and since $\varepsilon>0$ is arbitrary, $\beta(\operatorname{co}(S))=\beta(S)$. Thus $\beta$ satisfies property (K5).

If $S, T \in \mathcal{B}$ and $\varepsilon>0$, select $A_{1}, A_{2} \in \mathcal{A}$ and $B_{1}, B_{2} \in \mathcal{B}$ such that $S \subset A_{1}+B_{1}$ and $T \subset A_{2}+B_{2}$, with $\widetilde{\alpha}\left(A_{1}\right)+\alpha\left(B_{1}\right) \leq \beta(S)+\varepsilon$ and $\widetilde{\alpha}\left(A_{2}\right)+\alpha\left(B_{2}\right) \leq \beta(T)+\varepsilon$. 
Note that $A:=A_{1}+A_{2} \in \mathcal{A}$ and $B:=B_{1}+B_{2} \in \mathcal{B}$, and also that $S+T \subset A+B$. It follows that

$$
\begin{aligned}
\beta(S+T) & \leq \widetilde{\alpha}(A)+\alpha(B)=\widetilde{\alpha}\left(A_{1}+A_{2}\right)+\alpha\left(B_{1}+B_{2}\right) \\
& \leq \widetilde{\alpha}\left(A_{1}\right)+\alpha\left(B_{1}\right)+\widetilde{\alpha}\left(A_{2}\right)+\alpha\left(B_{2}\right) \leq \beta(S)+\beta(T)+2 \varepsilon .
\end{aligned}
$$

Since $\varepsilon>0$ is arbitrary, we see that $\beta(S+T) \leq \beta(S)+\beta(T)$, so $\beta$ satisfies property (K6).

The fact that $\beta$ satisfies property (K7), namely $\beta(\lambda S)=|\lambda| \beta(S)$ for all $S \in \mathcal{B}$ and $\lambda \in \mathbb{C}$, follows easily from the definition (12) of $\beta$ and the fact that $\widetilde{\alpha}$ and $\alpha$ satisfy property $(\mathrm{K} 7)$. Details are left to the reader.

If $S \in \mathcal{B}$, property (K2) implies that $\beta(S) \leq \beta(\bar{S})$. On the other hand, we have for any $\varepsilon>0$ that $\bar{S} \subset S+B_{\varepsilon}(0)$. Thus from the homogeneity of $\beta$ and from properties (K2) and (K6), we have that

$$
\beta(\bar{S}) \leq \beta(S)+\beta\left(B_{\varepsilon}(0)\right)=\beta(S)+\varepsilon \beta\left(B_{1}(0)\right) .
$$

This shows that $\beta(\bar{S}) \leq \beta(S)$ and proves property (K4).

If $T \in \mathcal{B}$ and $\bar{T}$ is compact, then $\beta(T)=0$ because $\beta(T) \leq \alpha(T)=0$. If $\bar{T}$ is compact and $S \in \mathcal{B}$, we claim that $\beta(S \cup T)=\beta(S)$, which certainly implies that property (K3) is satisfied. Property (K2) implies that $\beta(S) \leq \beta(S \cup T)$. To see the opposite inequality, select $x_{0} \in S$, define $\Gamma:=\left(T \cup\left\{x_{0}\right\}\right)+\left\{-x_{0}\right\}$, and note that $\bar{\Gamma}$ is compact and that $S \cup T \subset S+\Gamma$. Therefore

$$
\beta(S \cup T) \leq \beta(S+\Gamma) \leq \beta(S)+\beta(\Gamma) \leq \beta(S)+\alpha(\Gamma)=\beta(S),
$$

and so property (K3) holds.

Note that we do not claim that $\beta$ necessarily satisfies property (K8).

We now establish property (K1), which, along with the inequivalence of $\beta$ and $\alpha$, is the main point of our construction. First, as noted above, if $S \in \mathcal{B}$ and $\bar{S}$ is compact, then $\beta(S)=0$. Now suppose, conversely, that $S \in \mathcal{B}$ and $\beta(S)=0$. We have to show that $\alpha(S)=0$, which implies that $\bar{S}$ is compact. Given $\varepsilon>0$, equation (12) implies that there exist $A \in \mathcal{A}$ and $B \in \mathcal{B}$ with $S \subset A+B$ and $\widetilde{\alpha}(A)+\alpha(B)<\varepsilon$. Equation (11) implies that there exists an integer $N$ with $\alpha\left(\left(I-P_{N}\right) A\right)<\varepsilon$. It follows that $\left(I-P_{N}\right) S \subset\left(I-P_{N}\right) A+\left(I-P_{N}\right) B$ and so

$$
\begin{aligned}
\alpha\left(\left(I-P_{N}\right) S\right) & \leq \alpha\left(\left(I-P_{N}\right) A\right)+\alpha\left(\left(I-P_{N}\right) B\right) \\
& \leq \alpha\left(\left(I-P_{N}\right) A\right)+\alpha(B)+\alpha\left(P_{N} B\right) \\
& \leq \alpha\left(\left(I-P_{N}\right) A\right)+2 \alpha(B)<3 \varepsilon,
\end{aligned}
$$

where the second inequality in (10) has been used. Next, for $N$ as above, define $\kappa:=$ $a_{N} \varepsilon \leq \varepsilon$ and select $A^{\prime} \in \mathcal{A}$ and $B^{\prime} \in \mathcal{B}$ with $S \subset A^{\prime}+B^{\prime}$ such that $\widetilde{\alpha}\left(A^{\prime}\right)+\alpha\left(B^{\prime}\right)<\kappa$. The inequalities in (10) imply that $\widetilde{\alpha}\left(P_{N} A^{\prime}\right)<\kappa$ and $\alpha\left(P_{N} B^{\prime}\right)<\kappa$, and also $\alpha\left(P_{N} A^{\prime}\right) \leq a_{N}^{-1} \widetilde{\alpha}\left(P_{N} A^{\prime}\right)$. It follows that

$$
\begin{aligned}
\alpha\left(P_{N} S\right) & \leq \alpha\left(P_{N} A^{\prime}\right)+\alpha\left(P_{N} B^{\prime}\right) \\
& \leq \frac{\widetilde{\alpha}\left(P_{N} A^{\prime}\right)}{a_{N}}+\alpha\left(P_{N} B^{\prime}\right)<\left(\frac{1}{a_{N}}+1\right) \kappa \leq 2 \varepsilon .
\end{aligned}
$$

Thus

$$
\alpha(S) \leq \alpha\left(\left(I-P_{N}\right) S\right)+\alpha\left(P_{N} S\right)<3 \varepsilon+2 \varepsilon=5 \varepsilon,
$$

and since $\varepsilon>0$ is arbitrary, $\alpha(S)=0$. 
Finally, we show that $\beta$ is inequivalent to $\alpha$. For any $n \geq 1$ define

$$
Z_{n}:=\{z \in Z \mid z(i, j)=0 \text { for } i \neq n\}, \quad S_{n}:=\left\{z \in Z_{n} \mid\|z\|_{Z} \leq 1\right\} .
$$

Note that $\left(Z_{n},\|\cdot\|_{Z}\right)$ and $\left(Z_{n},\|\cdot\| \widetilde{Z}\right)$ are infinite dimensional Banach spaces, and in fact $\|z\|_{Z}=a_{n}^{-1}\|z\|_{\tilde{Z}}$ for every $z \in Z_{n}$. Thus Proposition $3 \operatorname{implies}$ that $\alpha\left(S_{n}\right)=2$, and also, since $S_{n}$ is also the closed ball of radius $a_{n}$ in the space $\left(Z_{n},\|\cdot\| \widetilde{Z}\right)$, Proposition 3 implies that $\widetilde{\alpha}\left(S_{n}\right)=2 a_{n}$. Further, $S_{n} \in \mathcal{A}$ and so we have that $\widetilde{\alpha}\left(S_{n}\right)=\beta\left(S_{n}\right)$, as noted earlier in this proof. Thus

$$
\lim _{n \rightarrow \infty}\left(\frac{\beta\left(S_{n}\right)}{\alpha\left(S_{n}\right)}\right)=\lim _{n \rightarrow \infty} a_{n}=0,
$$

and it follows that $\beta$ and $\alpha$ are inequivalent.

The above theorem suggests the following general question.

Open Question. Is it the case that for any infinite dimensional Banach space $(X,\|\cdot\|)$ there exists a homogeneous (possibly set-additive) MNC $\beta$ which is not equivalent to the Kuratowski MNC $\alpha$ on $X$ ?

In [12, we provide a partial answer to the above Open Question, by showing that for a large class of Banach spaces of interest in analysis, there does exist a homogeneous, set-additive MNC which is not equivalent to the Kuratowski MNC. In particular, this is verified for general Hilbert spaces; for the Banach spaces $L^{p}(\Omega, \Sigma, \mu)$, where $(\Omega, \Sigma, \mu)$ is a general measure space and $1 \leq p \leq \infty$; for $C(K)$, where $K$ is a compact Hausdorff space; and for the Sobolev space $W^{m, p}(\Omega)$, where $\Omega \subset \mathbb{R}^{n}$ is an open set. We believe, however, that an answer (positive or negative) to the Open Question is probably difficult and probably will involve techniques beyond those used in 12 .

Our next main result studies $\beta\left(\Lambda^{m}\right)$ and $\beta^{\#}(\Lambda)$ and the corresponding quantities for $\gamma$, for the MNC $\beta=\beta_{Z}$ in the proof of Theorem 7 and the homogeneous, setadditive MNC $\gamma=\gamma_{Z}$ derived from $\beta$ by Proposition 1 Recall the definitions and properties (1), (2), of $\beta\left(\Lambda^{m}\right)$ and $\beta^{\#}(\Lambda)$. We shall take $\Lambda$ to be a particular shift operator.

Theorem 8. With $Z=\ell^{p}(\mathbb{N} \times \mathbb{N})$, where $1 \leq p \leq \infty$, define $\Lambda: Z \rightarrow Z$ by $\Lambda z=x$, where $x(i, j)=z(i+1, j)$ for every $(i, j) \in \mathbb{N} \times \mathbb{N}$. Also fix a nonincreasing sequence $\left\{a_{n}\right\}_{n=1}^{\infty}$ as in the proof of Theorem 7 , with $\beta$ the homogeneous $M N C$ on $Z$ given by equation (12), and $\gamma$ the homogeneous, set-additive $M N C$ derived from $\beta$ as in Proposition 11. Then for every $m \geq 1$,

$$
\beta\left(\Lambda^{m}\right)=\gamma\left(\Lambda^{m}\right)=\mu_{m}:=\sup _{n \geq 1}\left(\frac{a_{n}}{a_{n+m}}\right),
$$

with the above formula serving as the definition of $\mu_{m} \in(1, \infty]$.

Remark. It is easily seen that $\left\|\Lambda^{m}\right\|_{\mathcal{L}(Z)}=1$ for every $m \geq 1$, so $\alpha\left(\Lambda^{m}\right) \leq 1$ by Lemma 4, where $\alpha$ is the Kuratowski MNC on $Z$. (Here and below we let $\|\cdot\|_{\mathcal{L}(X)}$ denote the operator norm associated to a space $X$.) In fact one easily sees that $\alpha\left(\Lambda^{m}\right)=1$ for every $m$, and so by earlier remarks we have that $\alpha^{\#}(\Lambda)=\rho(\Lambda)=1$.

Proof of Theorem 8 . Let $m \geq 1$ be an integer which will be fixed for the remainder of the proof. Generally, we shall use the notation and constructions from the proof of Theorem 7 assuming as well that $p<\infty$. 
Let $S \in \mathcal{B}$ with $S=\bigcup_{i=1}^{n} S_{i}$ for some $S_{i}$ where $n<\infty$. Then $\Lambda^{m} S=\bigcup_{i=1}^{n} \Lambda^{m} S_{i}$ and so

$$
\gamma\left(\Lambda^{m} S\right) \leq \max _{1 \leq i \leq n} \beta\left(\Lambda^{m} S_{i}\right) \leq \beta\left(\Lambda^{m}\right) \max _{1 \leq i \leq n} \beta\left(S_{i}\right)
$$

from the definition (7) of $\gamma$ and from Lemma 4. As the above inequalities are valid for every $S_{i}$, it follows that $\gamma\left(\Lambda^{m} S\right) \leq \beta\left(\Lambda^{m}\right) \gamma(S)$ and thus $\gamma\left(\Lambda^{m}\right) \leq \beta\left(\Lambda^{m}\right)$.

Next suppose that $S \in \mathcal{A}$, again with $S=\bigcup_{i=1}^{n} S_{i}$ for some $S_{i}$. Then $S_{i} \in \mathcal{A}$ for each $i$, and $\beta(S)=\widetilde{\alpha}(S)$ and $\beta\left(S_{i}\right)=\widetilde{\alpha}\left(S_{i}\right)$, as noted in the proof of Theorem 7 Thus

$$
\beta(S)=\widetilde{\alpha}(S)=\max _{1 \leq i \leq n} \widetilde{\alpha}\left(S_{i}\right)=\max _{1 \leq i \leq n} \beta\left(S_{i}\right)
$$

from the set-additivity of $\widetilde{\alpha}$, and this implies that $\gamma(S)=\beta(S)$.

Now recall the set $S_{n} \subset Z$ as in (13) and the fact, noted in the proof of Theorem 7 that $\beta\left(S_{n}\right)=2 a_{n}$. Certainly $S_{n} \in \mathcal{A}$, and so also $\gamma\left(S_{n}\right)=2 a_{n}$. Observing that $\Lambda^{m} S_{n+m}=S_{n}$ for every $n \geq 1$, we have that $\gamma\left(\Lambda^{m} S_{n+m}\right)=\left(\frac{a_{n}}{a_{n+m}}\right) \gamma\left(S_{n+m}\right)$ and therefore $\gamma\left(\Lambda^{m}\right) \geq \frac{a_{n}}{a_{n+m}}$. Taking the supremum over $n \geq 1$, we conclude that $\gamma\left(\Lambda^{m}\right) \geq \mu_{m}$. It remains to prove that $\beta\left(\Lambda^{m}\right) \leq \mu_{m}$. If $\mu_{m}=\infty$ we are done, so assume for the remainder of the proof that $\mu_{m}<\infty$.

Recall the Banach space $(\widetilde{Z},\|\cdot\| \widetilde{Z})$ in the proof of Theorem 7 . For any $z \in \widetilde{Z}$ we have that

$$
\begin{aligned}
\left\|\Lambda^{m} z\right\|_{\widetilde{Z}} & =\left(\sum_{i=m+1}^{\infty} a_{i-m}^{p} \sum_{j=1}^{\infty}|z(i, j)|^{p}\right)^{1 / p} \\
& \leq\left(\sum_{i=m+1}^{\infty} \mu_{m}^{p} a_{i}^{p} \sum_{j=1}^{\infty}|z(i, j)|^{p}\right)^{1 / p} \leq \mu_{m}\|z\|_{\widetilde{Z}}
\end{aligned}
$$

and it follows that $\Lambda^{m} \widetilde{Z} \subset \widetilde{Z}$ and $\left\|\Lambda^{m}\right\|_{\mathcal{L}(\widetilde{Z})} \leq \mu_{m}$. On the other hand, let $n>m$ and take any $z \in Z_{n}$, with $Z_{n}$ as in (13). Then $\Lambda^{m} z \in Z_{n-m}$ and so $z, \Lambda^{m} z \in \widetilde{Z}$ with

$$
\left\|\Lambda^{m} z\right\|_{\tilde{Z}}=a_{n-m}\left(\sum_{j=1}^{\infty}|z(n, j)|^{p}\right)^{1 / p}=\left(\frac{a_{n-m}}{a_{n}}\right)\|z\|_{\tilde{Z}} .
$$

It follows that $\left\|\Lambda^{m}\right\|_{\mathcal{L}(\widetilde{Z})} \geq \mu_{m}$ and thus

$$
\left\|\Lambda^{m}\right\|_{\mathcal{L}(\widetilde{Z})}=\mu_{m} .
$$

Now take any $S \in \mathcal{B}$ and $\varepsilon>0$. Then there exist $A \in \mathcal{A}$ and $B \in \mathcal{B}$ so that $S \subset A+B$ and

$$
\beta(S) \leq \widetilde{\alpha}(A)+\alpha(B)<\beta(S)+\varepsilon,
$$

by the definition (12) of $\beta$. The reader can verify that $\alpha\left(\left(I-P_{n}\right) \Lambda^{m} A\right)=\alpha((I-$ $\left.P_{n+m}\right) A$ ), which implies that $\Lambda^{m} A \in \mathcal{A}$. We have $\Lambda^{m} S \subset \Lambda^{m} A+\Lambda^{m} B$ and also $\mu_{m} \geq 1$, so it follows from Lemma 4. from (14), and because $\left\|\Lambda^{m}\right\|_{\mathcal{L}(Z)}=1$ that

$$
\begin{aligned}
& \beta\left(\Lambda^{m} S\right) \leq \widetilde{\alpha}\left(\Lambda^{m} A\right)+\alpha\left(\Lambda^{m} B\right) \\
& \leq\left\|\Lambda^{m}\right\|_{\mathcal{L}(\widetilde{Z})} \widetilde{\alpha}(A)+\left\|\Lambda^{m}\right\|_{\mathcal{L}(Z)} \alpha(B) \\
& =\mu_{m} \widetilde{\alpha}(A)+\alpha(B) \leq \mu_{m}(\beta(S)+\varepsilon) .
\end{aligned}
$$

We conclude that $\beta\left(\Lambda^{m}\right) \leq \mu_{m}$, as desired; hence $\beta\left(\Lambda^{m}\right)=\gamma\left(\Lambda^{m}\right)=\mu_{m}$, as claimed. 
Remark. Any value $s \in(1, \infty]$ for the quantity $\beta^{\#}(\Lambda)$ can be obtained by a suitable choice of the sequence $\left\{a_{n}\right\}_{n=1}^{\infty}$ in the above construction. If $s \in(1, \infty)$, then taking $a_{n}=s^{-n}$ gives $\beta\left(\Lambda^{m}\right)=\mu_{m}=s^{m}$, and hence $\beta^{\#}(\Lambda)=s$. If $s=\infty$, then taking, for example, $a_{n}=n^{-n}$ gives $\beta\left(\Lambda^{m}\right)=\mu_{m}=\infty$ for every $m$, and hence $\beta^{\#}(\Lambda)=\infty$.

While the above construction has been carried out for the space $\ell^{p}(\mathbb{N} \times \mathbb{N})$, where $1 \leq p \leq \infty$, with the aid of results in [12] analogs of Theorem 8 can be proved for a variety of infinite dimensional Banach spaces which arise naturally in analysis.

We return again to the general case. Let $(X,\|\cdot\|)$ be any complex, infinite dimensional Banach space, $\beta$ an arbitrary homogeneous MNC on $X$, and $L: X \rightarrow$ $X$ any bounded linear map. There are several inequivalent definitions of $\operatorname{ess}(L)$, the essential spectrum of $L$, and all definitions actually apply when $L: \mathcal{D}(L) \subset X \rightarrow X$ is closed and densely defined. For example, F.E. Browder 5 defines ess $(L)$ to be the set of $\lambda \in \mathbb{C}$ such that (a) $\lambda$ is an accumulation point of $\sigma(L)$, the spectrum of $L$, or that (b) $\mathcal{R}(\lambda I-L)$, the range of $\lambda I-L$, is not closed, or that (c) $\bigcup_{i=1}^{\infty} \mathcal{N}\left((\lambda I-L)^{i}\right)$ is infinite dimensional, where $\mathcal{N}(B)$ denotes the null space of a linear map $B$. Another possible definition is $\operatorname{ess}(L)=\{\lambda \in \mathbb{C} \mid \lambda I-L$ is not Fredholm of index 0$\}$. F. Wolf [19] defines ess $(L)=\{\lambda \in \mathbb{C} \mid \lambda I-L$ is not Fredholm $\}$, and T. Kato [9] defines ess $(L)=\{\lambda \in \mathbb{C} \mid \lambda I-L$ is not semi-Fredholm $\}$. Simple examples involving shift operators on $\ell^{2}(\mathbb{N})$ show that these definitions are not equivalent. However, by using classical results of Gohberg and Krein $[8$ and index theory for semi-Fredholm operators (see [9]), one can prove that for all definitions, $\operatorname{ess}(L)$ is nonempty and that

$$
\rho(L):=\sup \{|\lambda| \mid \lambda \in \operatorname{ess}(L)\}
$$

is the same for all definitions of $\operatorname{ess}(L)$. If $|\lambda|>\rho(L)$ and $\lambda \in \sigma(L)$, then $\lambda$ is an eigenvalue of $L$ of finite algebraic multiplicity, $\lambda$ is an isolated point of $\sigma(L)$, and $\lambda I-L$ is Fredholm of index 0 .

Now let $\alpha$ denote the Kuratowski MNC on $X$ and define $\eta$, the ball measure of noncompactness on $\mathrm{X}$, by

$$
\eta(S):=\inf \left\{r>0 \mid S \subset \bigcup_{i=1}^{n} B_{r}\left(x_{i}\right) \text { for some } x_{i} \in X, \text { for } 1 \leq n<\infty\right\},
$$

with $B_{r}(x)$ as in (4). It is well-known that $\eta$ is a homogeneous, set-additive MNC and that

$$
\frac{\alpha(S)}{2} \leq \eta(S) \leq \alpha(S)
$$

for every $S \in \mathcal{B}(X)$. If $L: X \rightarrow X$ is a bounded linear map, it is also known (see Lemma 1 in [14]) that

$$
\eta\left(L^{m}\right)=\eta\left(L^{m} B_{1}(0)\right) .
$$

It follows from equations (16) and (17) and earlier remarks that

$$
\rho(L)=\eta^{\#}(L)=\lim _{m \rightarrow \infty} \eta\left(L^{m} B_{1}(0)\right)^{1 / m}=\lim _{m \rightarrow \infty} \alpha\left(L^{m} B_{1}(0)\right)^{1 / m}=\alpha^{*}(L),
$$

where $\rho(L)$ is as in (15) and where we recall that $\beta^{*}(L)$, for any homogeneous MNC $\beta$, is given by (3). As any such $\beta$ is dominated by $\alpha$ by Proposition 2 it follows from (18) that

$$
\beta^{*}(L) \leq \alpha^{*}(L)=\rho(L)
$$


We claim that $\beta^{*}(L)=\rho(L)$. To prove this we shall use an old result of Yood 20] and some facts about semi-Fredholm operators (see [9]). In the following lemma, recall that a map $f$ from a topological space $U$ to a topological space $V$ is called proper if $f^{-1}(K)$ is compact (possibly empty) for every compact $K \subset V$.

Lemma 9 (Yood [20]). Let $X$ and $Y$ be Banach spaces (real or complex) and $L: X \rightarrow Y$ a bounded linear map. Then the map $L \mid S: S \rightarrow Y$ is proper for every closed, bounded $S \subset X$ if and only if $\mathcal{N}(L)$, the null space of $L$, is finite dimensional, and $\mathcal{R}(L)$, the range of $L$, is closed.

Theorem 10. Let $X$ be a complex Banach space, $L: X \rightarrow X$ a bounded linear map, and $\beta$ any homogeneous $M N C$ on $X$. Then

$$
\beta^{*}(L)=\rho(L),
$$

where $\beta^{*}(L)$ is given by equation (3) and $\rho(L)$ by equation (15). If instead $X$ is a real Banach space, then

$$
\beta^{*}(L)=\rho(\widehat{L}),
$$

where $\widehat{L}: \widehat{X} \rightarrow \widehat{X}$ is the complexification of $L$ and $\widehat{X}$ is the complexification of $X$.

Proof. First suppose that $X$ is a complex Banach space. Let $r>0$ and $|\lambda|>\beta^{*}(L)$, and denote $L_{\lambda}:=\lambda^{-1} L$. Then by equation (3),

$$
\lim _{m \rightarrow \infty} \beta\left(L_{\lambda}^{m} B_{r}(0)\right)=\lim _{m \rightarrow \infty} r \beta\left(L_{|\lambda|}^{m} B_{1}(0)\right)=0 .
$$

Let $Q_{r}:=\overline{B_{r}(0)}$. We claim that $\left(I-L_{\lambda}\right) \mid Q_{r}$ is proper, equivalently, that $(\lambda I-L) \mid Q_{r}$ is proper. As $r>0$ is arbitrary, this implies that $(\lambda I-L) \mid S$ is proper for every closed, bounded $S \subset X$. To prove our claim, let $K \subset X$ be compact and let $T:=\left\{x \in Q_{r} \mid\left(I-L_{\lambda}\right) x \in K\right\}$. The set $T$ is closed, by continuity. If $x \in T$, then $x=L_{\lambda} x+y$ for some $y \in K$, and it follows for all $m \geq 1$ that $x=L_{\lambda}^{m} x+\sum_{i=0}^{m-1} L_{\lambda}^{i} y$. This implies that

$$
T \subset L_{\lambda}^{m} T+\left(\sum_{i=0}^{m-1} L_{\lambda}^{i}\right) K \subset L_{\lambda}^{m} Q_{r}+K_{m}
$$

where $K_{m}:=\left(\sum_{i=0}^{m-1} L_{\lambda}^{i}\right) K$ is compact. It follows from (22) that

$$
\beta(T) \leq \beta\left(L_{\lambda}^{m} Q_{r}\right)+\beta\left(K_{m}\right)=\beta\left(L_{\lambda}^{m} Q_{r}\right) \leq \beta\left(\overline{L_{\lambda}^{m} B_{r}(0)}\right)=\beta\left(L_{\lambda}^{m} B_{r}(0)\right),
$$

and with (21) it follows that $\beta(T)=0$. Thus $T$ is compact. Yood's lemma now implies that $\mathcal{N}(\lambda I-L)$ is finite dimensional and $\mathcal{R}(\lambda I-L)$ is closed, that is, $\lambda I-L$ is a semi-Fredholm operator with index $i(\lambda I-L):=\operatorname{dim}(\mathcal{N}(\lambda I-L))-$ $\operatorname{codim}(\mathcal{R}(\lambda I-L))<\infty$. Moreover, the value of $i(\lambda I-L)$ is independent of such a $\lambda$ due to the continuity of the index of semi-Fredholm operators. As $\lambda I-L$ is invertible for $|\lambda|>\|L\|$, this value is $i(\lambda I-L)=0$. Thus $\lambda I-L$ is Fredholm of index 0 for all $\lambda$ with $|\lambda|>\beta^{*}(L)$. Using Wolf's definition of ess $(L)$ we have that $\rho(L) \leq \beta^{*}(L)$; thus $\rho(L)=\beta^{*}(L)$ from (19).

If $X$ is a real Banach space, then (20) follows from (6) and the surrounding remark.

Lastly, we prove the following result, which was discussed in a remark above. 
Proposition 11. Let $X$ be a real Banach space, let $\alpha$ denote the Kuratowski MNC on $X$, and let $\widehat{\alpha}$ denote its complexification, as in (5). Then $\widehat{\alpha}$ is also the Kuratowski $M N C$ on $\widehat{X}$.

Proof. With $\widehat{\alpha}$ denoting the complexification of $\alpha$ as in the statement of the proposition, let $\bar{\alpha}$ denote the Kuratowski MNC on $\widehat{X}$. We must show that $\widehat{\alpha}=\bar{\alpha}$. First observe that if $\widehat{S} \subset \widehat{X}$ is any bounded set, then $\operatorname{diam}\left(e^{i \theta} \widehat{S}\right)=\operatorname{diam}(\widehat{S})$ and $\operatorname{diam}(\operatorname{Re}(\widehat{S})) \leq \operatorname{diam}(\widehat{S})$; hence

$$
\operatorname{diam}\left(\operatorname{Re}\left(e^{i \theta} \widehat{S}\right)\right) \leq \operatorname{diam}(\widehat{S}),
$$

for any $\theta \in \mathbb{R}$. Now with such an $\widehat{S}$ fixed, denote $\bar{a}=\bar{\alpha}(\widehat{S})$ and let $\varepsilon>0$. Then $\widehat{S}=\bigcup_{j=1}^{n} \widehat{S}_{j}$ for some sets $\widehat{S}_{1}, \widehat{S}_{2}, \ldots, \widehat{S}_{n} \subset \widehat{X}$, each with $\operatorname{diam}\left(\widehat{S}_{j}\right) \leq \bar{a}+\varepsilon$. For any $\theta \in \mathbb{R}$ we have that $\operatorname{Re}\left(e^{i \theta} \widehat{S}\right)=\bigcup_{j=1}^{n} \operatorname{Re}\left(e^{i \theta} \widehat{S}_{j}\right)$, and as $\operatorname{diam}\left(\operatorname{Re}\left(e^{i \theta} \widehat{S}_{j}\right)\right) \leq \bar{a}+\varepsilon$, it follows that $\alpha\left(\operatorname{Re}\left(e^{i \theta} \widehat{S}\right)\right) \leq \bar{a}+\varepsilon$. Taking the supremum over $\theta$ and letting $\varepsilon \rightarrow 0$ now gives $\widehat{\alpha}(\widehat{S}) \leq \bar{a}=\bar{\alpha}(\widehat{S})$.

Now denote $\widehat{a}=\widehat{\alpha}(\widehat{S})$. Then $\alpha\left(\operatorname{Re}\left(e^{i \theta} \widehat{S}\right)\right) \leq \widehat{a}$ for every $\theta$. Fix $m>0$ and let $\theta_{k}=\frac{2 \pi k}{m}$ for $1 \leq k \leq m$. Also fix $\varepsilon>0$. Then for each $k$ in the above range there exist sets $S_{k, j} \subset X$ for $1 \leq j \leq n_{k}<\infty$ such that $\operatorname{Re}\left(e^{i \theta_{k}} \widehat{S}\right)=\bigcup_{j=1}^{n_{k}} S_{k, j}$ with

$$
\operatorname{diam}\left(S_{k, j}\right) \leq \alpha\left(\operatorname{Re}\left(e^{i \theta_{k}} \widehat{S}\right)\right)+\varepsilon \leq \widehat{a}+\varepsilon .
$$

Now define $\widehat{S}_{k, j}=\left\{x \in \widehat{S} \mid \operatorname{Re}\left(e^{i \theta_{k}} x\right) \in S_{k, j}\right\}$, so clearly $\widehat{S}=\bigcup_{j=1}^{n_{k}} \widehat{S}_{k, j}$ for every $k$. Now consider all sequences $\sigma=\left(j_{1}, j_{2}, \ldots, j_{m}\right)$ where $1 \leq j_{k} \leq n_{k}$, and for each such $\sigma$ let $\widehat{T}_{\sigma}=\bigcap_{k=1}^{m} \widehat{S}_{k, j_{k}}$. Then $\widehat{S}=\bigcup \widehat{T}_{\sigma}$, where the union is taken over all possible such sequences $\sigma$, of which there are finitely many. We wish to obtain an upper bound for the diameter of $\widehat{T}_{\sigma}$ for each $\sigma$. Fixing $\sigma=\left(j_{1}, j_{2}, \ldots, j_{m}\right)$, let $x, y \in \widehat{T}_{\sigma}$. For any $k$ with $1 \leq k \leq m$ we have that $x, y \in \widehat{S}_{k, j_{k}}$, and therefore $\operatorname{Re}\left(e^{i \theta_{k}} x\right), \operatorname{Re}\left(e^{i \theta_{k}} y\right) \in S_{k, j_{k}}$. Thus

$$
\left\|\operatorname{Re}\left(e^{i \theta_{k}}(x-y)\right)\right\|=\left\|\operatorname{Re}\left(e^{i \theta_{k}} x\right)-\operatorname{Re}\left(e^{i \theta_{k}} y\right)\right\| \leq \operatorname{diam}\left(S_{k, j_{k}}\right) \leq \widehat{a}+\varepsilon .
$$

Denoting $x-y=u+i v$, where $u, v \in X$, this can be written as

$$
\left\|\left(\cos \theta_{k}\right) u-\left(\sin \theta_{k}\right) v\right\| \leq \widehat{a}+\varepsilon .
$$

Now for any $\theta \in[0,2 \pi]$, there exists $k$ such that $\left|\theta-\theta_{k}\right| \leq \frac{2 \pi}{m}$. Then

$$
\begin{aligned}
& \|(\cos \theta) u-(\sin \theta) v\| \\
& \quad \leq\left\|\left(\cos \theta_{k}\right) u-\left(\sin \theta_{k}\right) v\right\|+\left\|\left(\cos \theta-\cos \theta_{k}\right) u-\left(\sin \theta-\sin \theta_{k}\right) v\right\| \\
& \quad \leq\left\|\left(\cos \theta_{k}\right) u-\left(\sin \theta_{k}\right) v\right\|+\frac{2 \pi}{m}\|u\|+\frac{2 \pi}{m}\|v\| \leq \widehat{a}+\varepsilon+\frac{4 \pi}{m}\|x-y\| .
\end{aligned}
$$

Taking the supremum over $\theta$ in the first term above gives $\|u-i v\|$, and upon noting that $\|u-i v\|=\|u+i v\|=\|x-y\|$ we obtain

$$
\|x-y\| \leq \widehat{a}+\varepsilon+\frac{4 \pi}{m}\|x-y\| \leq \widehat{a}+\varepsilon+\frac{4 \pi}{m} \operatorname{diam}(\widehat{S}) .
$$

As $x, y \in T_{\sigma}$ are arbitrary, this gives an upper bound for $\operatorname{diam}\left(T_{\sigma}\right)$ and thus an upper bound

$$
\bar{\alpha}(\widehat{S}) \leq \widehat{a}+\varepsilon+\frac{4 \pi}{m} \operatorname{diam}(\widehat{S})
$$


for the Kuratowski MNC of $\widehat{S}$. As $\varepsilon$ and $m$ are arbitrary, it follows that $\bar{\alpha}(\widehat{S}) \leq$ $\widehat{a}=\widehat{\alpha}(\widehat{S})$. With this, the proposition is proved.

\section{REFERENCES}

[1] R.R. Akhmerov, M.I. Kamenskij, A.S. Potapov, A.E. Rodkina, and B.N. Sadovskij, Measures of Noncompactness and Condensing Operators (in Russian), Nauka, Novosibirsk, 1986; English translation: Birkhäuser Verlag, Basel, 1992. MR 1153247 (92k:47104)

[2] J. Appell, Measures of noncompactness, condensing operators and fixed points: An application-oriented survey, Fixed Point Theory 6 (2005), pp. 157-229. MR 2196709 (2006h:47121)

[3] J.M. Ayerbe Toledano, T. Dominguez Benavides, and G. López Acedo, Measures of Noncompactness in Metric Fixed Point Theory, Birkhäuser Verlag, Basel, 1997. MR.1483889 (99e:47070)

[4] J. Banaś and K. Goebel, Measures of Noncompactness in Banach Spaces, Marcel Dekker, New York, 1980. MR591679 (82f:47066)

[5] F.E. Browder, On the spectral theory of elliptic differential operators, Math. Ann. 142 (1961), pp. 22-130. MR0209909 (35:804)

[6] G. Darbo, Punti uniti in trasformazioni a condominio non compatto, Rend. Sem. Mat. Univ. Padova 24 (1955), pp. 84-92. MR0070164(16:1140f)

[7] M. Furi and A. Vignoli, On a property of the unit sphere in a linear normed space, Bull. Acad. Polon. Sci. Sér. Sci. Math. Astronom. Phys. 18 (1970), pp. 333-334. MR0264373 (41:8969)

[8] I. Gohberg and M.G. Krein, The basic propositions on defect numbers, root numbers and indices of linear operators, Amer. Math. Soc. Translations, Series 2, vol. 13 (1960), pp. 185264. MR0113146 (22:3984)

[9] T. Kato, Perturbation Theory for Linear Operators, Springer-Verlag, New York, 1966. MR0203473 (34:3324)

[10] K. Kuratowski, Sur les espaces complets, Fund. Math. 15 (1930), pp. 301-309.

[11] J. Mallet-Paret and R.D. Nussbaum, Eigenvalues for a class of homogeneous cone maps arising from max-plus operators, Discrete and Continuous Dynamical Systems 8 (2002), pp. 519-562. MR.1897866 (2003c:47088)

[12] J. Mallet-Paret and R.D. Nussbaum, Inequivalent measures of noncompactness, Ann. Mat. Pura Appl., to appear.

[13] J. Mallet-Paret and R.D. Nussbaum, Generalizing the Krein-Rutman theorem, measures of noncompactness and the fixed point index, J. Fixed Point Theory and Appl. 7 (2010), pp. 103-143.

[14] R.D. Nussbaum, The radius of the essential spectrum, Duke Math. J. 37 (1970), pp. 473-478. MR0264434(41:9028)

[15] R.D. Nussbaum, A generalization of the Ascoli theorem and an application to functional differential equations, J. Math. Anal. Appl. 35 (1971), pp. 600-610. MR0289898(44:7085)

[16] R.D. Nussbaum, The fixed point index for local condensing maps, Ann. Mat. Pura Appl. 89 (1971), pp. 217-258. MR0312341 (47:903)

[17] R.D. Nussbaum, Eigenvalues of nonlinear operators and the linear Krein-Rutman theorem, in Fixed Point Theory, Springer Lecture Notes in Math., vol. 886, Springer-Verlag, Berlin, 1981, pp. 309-331. MR643014 (83b:47068)

[18] B.N. Sadovskij, Limit-compact and condensing operators, Uspekhi Mat. Nauk 27 (1972), pp. 81-146 (in Russian). MR0428132 (55:1161)

[19] F. Wolf, On the essential spectrum of partial differential boundary problems, Comm. Pure Appl. Math. 12 (1959), pp. 211-228. MR0107750(21:6472)

[20] B. Yood, Properties of linear transformations preserved under addition of a completely continuous transformation, Duke Math. J. 18 (1951), pp. 599-612. MR0044020(13:355f)

Division of Applied Mathematics, Brown University, Providence, Rhode Island 02912

E-mail address: jmp@dam.brown.edu

Department of Mathematics, Rutgers University, Piscataway, New Jersey 08854

E-mail address: nussbaum@math.rutgers.edu 